\title{
Factor XIIla-positive dermal dendritic cells and HLA-DR expression in radial versus vertical growth-phase melanomas
}

Fifty nevomelanocytic lesions, including 10 typical compound nevi and 20 radial and 20 vertical growth-phase melanomas, were evaluated for factor XIIIa and HLA-DR (LN3) expression within dermal dendritic cells (DDCs) or dermal dendrocytes to determine if DDCs proliferate and/or participate as possible antigenpresenting cells in the local tissue response to benign and malignant nevomelanocytic lesions. There was no statistical difference in factor XIIIa staining of DDCs between nevi and radial or vertical growth-phase melanomas, suggesting that DDCs do not significantly proliferate in nevomelanocytic lesions. However, studies to determine proliferation rate, apoptosis, and influences of local mediators on cell growth and/or recruitment were not done. HLA-DR staining by DDCs was significantly increased $(\mathrm{p}<0.001)$ in both melanoma groups when compared to compound nevi, but did not significantly differ between radial and vertical growthphase melanomas. The intensity of HLA-DR expression appeared to correlate with the presence or absence of lymphocytic inflammation; HLA-DR intensity was judged greater in melanomas characterized by a brisk and infiltrative lymphocytic host response. We propose that DDCs may participate in the dermal immune response to invasive melanomas, probably as antigen-presenting cells to skin-associated lymphocytes.

Fullen DR, Headington JT. Factor XIIIa-positive dermal dendritic cells and HLA-DR expression in radial versus vertical growthphase melanomas.

J Cutan Pathol 1998: 25: 553-558. ㅇ Munksgaard 1998.

\author{
Douglas R. Fullen and \\ John T. Headington
}

Department of Pathology, University of Michigan Hospitals, Ann Arbor, Michigan, USA

\begin{abstract}
Douglas R. Fullen, M.D., Department of Pathology, Cornell University Weill Medical Center, 1300 York Avenue, F-309, New York, NY 10021, USA. Tel: 212-746-6434, Fax: 212-746-8570
\end{abstract}

Accepted July 29, 1998
Dermal dendritic cells (DDCs) or dermal dendrocytes are normal resident cells of the reticular dermal interstitium, occurring predominantly perivascularly and within the papillary dermis. They are characterized by their highly dendritic morphology (1). Although the origin of the DDC remains a topic of debate, the preponderance of evidence from immunohistochemistry and immunofluorescence studies supports their derivation from bone marrow progenitor cells $(2,3)$.

Most DDCs express cytoplasmic factor XIIIa, a protransglutaminase involved in hemostasis (4-6). In addition to their putative role in wound healing, DDCs are capable of phagocytosis of hemosiderin, melanin, and exogenous pigments, such as are present in tattoos $(1,4,7)$.
DDCs proliferate in a variety of inflammatory and neoplastic processes. In inflammatory dermatoses, such as psoriasis and atopic eczema, an increase in DDCs associated with a lymphocytic infiltrate was previously reported (3). A proliferation of factor XIIIa-positive DDCs is the sine qua non of the dermatofibroma, and is also seen in fibrous papules of the face $(8,9)$. Moreover, DDCs are readily identified at the periphery of Kaposi's sarcomas (10). Denton et al. evaluated the distribution of factor XIIIa-positive DDCs in distinguishing nodular melanomas from Spitz nevi (11). They demonstrated that DDCs were either diffusely distributed within the tumor or primarily localized to the periphery, and that the pattern of distribution could not be used to distinguish between these two entities. 
A proposed antigen-presenting role for DDCs is based on their proliferation and enhanced expression of HLA-DR, a class II, nonconstitutively expressed major histocompatibility antigen, in intradermal delayed hypersensitivity reactions (1) and as potent stimulators of allogeneic mixed lymphocyte reactions in vitro (12). Although HLA-DR expression in melanomas has been extensively studied, the primary focus has been on antigen expression in melanoma cells (13-19). A few groups have evaluated HLA-DR expression by dendritic cells (Langerhans cells) residing within the dermis $(20,21)$; however, to our knowledge, differences in HLA-DR expression by factor XIIIa-positive DDCs in radial versus vertical growth-phase melanomas has not been done.

In this study, we semiquantitatively evaluated factor XIIIa expression by DDCs in benign and malignant nevomelanocytic lesions to determine if there was a hyperplasia of DDCs and, if so, whether there specifically was a difference between radial and vertical growth-phase melanomas. Moreover, we assessed HLA-DR expression by DDCs to delineate a possible immunologic role as antigen-presenting cells in the progression of malignant melanomas.

\section{Material and methods}

Case selection

Fifty cases, 20 radial and 20 vertical growth-phase melanomas and 10 typical compound nevi, were obtained through the Melanoma Clinic registry and the SNOMED retrieval program of the Pathology Data Systems of the University of Michigan Medical Center. Cases were excluded if they were re-excisions of a previously diagnosed melanoma or compound nevus.

Radial were compared to vertical growth-phase melanomas for sex, age, body site, presence or absence of regression, and sun exposure. There were more males than females in the radial growthphase group (14 vs. 6), as opposed to slightly more females than males in the vertical growth-phase group (11 vs. 9). The mean age was not appreciably different between the two groups (radial, 56 years; vertical, 61 years). Melanomas were separated into sites as follows: head and neck (radial, 4; vertical, 5), trunk (radial, 12; vertical, 8), extremities (radial, 3; vertical, 7), and site not provided (radial, 1). Presence of regression did not differ between the two groups (radial, 5; vertical, 4). In cases with regression, it was either focal (radial, 1; vertical, 2), or diffuse (radial, 4; vertical, 2). The degree of sun exposure was determined based on the degree of elastosis seen on the original hematoxylin and eosin stained slides. There was no sig- nificant difference between radial or vertical growth-phase melanomas with respect to presence (radial, 14; vertical, 15), or degree of elastosis.

\section{Immunoperoxidase methods}

Four- $\mu \mathrm{m}$ sections of formalin-fixed, paraffin-embedded tissue were prepared on plus slides (Baxter Scientific). In the vast majority of cases, sequential slides were prepared at approximately 4$\mu \mathrm{m}$ intervals and consecutively stained with the primary antibodies in question.

\section{Single labeling}

The slides were deparaffinized in xylene and ethanol. Endogenous peroxidases were blocked with hydrogen peroxide. Pretreatment with protease was done for factor XIIIa and CDla stains, and microwave for CD34 (HPCA-1/2) stains; no pretreatment was done for HLA-DR (LN3). The tissues were stained with primary antibody followed by a biotinylated, polyspecific secondary antibody and an avidin-biotin horseradish peroxidase amplification system using an automated (Ventana) method. Each case was stained with a 1:800 dilution of rabbit polyclonal antibody to factor XIIIa (Becton-Dickinson), an undiluted mouse monoclonal antibody to HLA-DR (BioGenex), an undiluted mouse monoclonal antibody to CDla (Immunotech), and a 1:10 dilution of mouse monoclonal antibody to CD34 (Becton-Dickinson). The peroxidase was visualized with 3',3'-diaminobenzadine (DAB). All slides were counterstained with Mayer's hematoxylin. Each antibody was applied to all 50 cases in a single run to avoid interbatch variability.

\section{Double labeling}

Six cases, three radial and three vertical growthphase melanomas, were double labeled with HLADR vs. factor XIIIa, HLA-DR vs. CD34, and CD34 vs. factor XIIIa. The slides were deparaffinized as above. Endogenous peroxidases were blocked with hydrogen peroxide. Microwave pretreatment was performed for optimal antigen retrieval of HLA-DR, factor XIIIa, and CD34, as determined by single stain control runs using different forms of pretreatment, i.e. no pretreatment, enzyme pretreatment, or microwave pretreatment. The tissues were stained with the first primary antibody (HLADR for HLA-DR vs. factor XIIIa or HLA-DR vs. CD34, or CD34 for CD34 vs. factor XIIIa) at the same titers as above, followed by a biotinylated, polyspecific (anti-mouse and anti-rabbit) secondary antibody and an avidin-biotin horseradish 
Table 1. Results of factor XIlla staining of DDCs.

\begin{tabular}{lcccc}
\hline Diagnosis & \multicolumn{4}{c}{ Intensity of staining } \\
\cline { 2 - 5 } & 0 & $1+$ & $2+$ & $3+$ \\
\hline Compound nevi & 6 & 4 & 0 & 0 \\
Radial growth-phase melanomas & 11 & 9 & 0 & 0 \\
Vertical growth-phase melanomas & 8 & 9 & 3 & 0 \\
\hline
\end{tabular}

Table 2. Results of HLA-DR staining of DDCs.

\begin{tabular}{lcccc}
\hline Diagnosis & \multicolumn{4}{c}{ Intensity of staining } \\
\cline { 2 - 5 } & 0 & $1+$ & $2+$ & $3+$ \\
\hline Compound nevi & 8 & 2 & 0 & 0 \\
Radial growth-phase melanomas & 3 & 10 & 7 & 0 \\
Vertical growth-phase melanomas & 3 & 11 & 6 & 0 \\
\hline
\end{tabular}

peroxidase amplification system by an automated (Ventana) method. The peroxidase reaction product was detected using DAB. The slides were then subjected to the second primary antibody (factor XIIIa or CD34 for HLA-DR, or factor XIIIa for CD34) followed by a biotinylated, polyspecific (anti-mouse and anti-rabbit) secondary antibody and an avidin-biotin alkaline phosphatase amplification system also using an automated (Ventana) system. The alkaline phosphatase reaction product was detected using fast red. All slides were counterstained with Mayer's hematoxylin.

\section{Interpretation of stains}

Factor XIIIa, HLA-DR, CD-34, and CDla slides were interpreted for staining intensity and scored semiquantitatively on a scale of $0-3+$. The scoring system for staining intensity was as follows: 0 $($ none $)=$ rare to no cells staining; $1+($ slight $)=$ low density, patchy staining; $2+($ moderate $)=$ moder ate density, focal or confluent paralesional staining; and $3+($ marked $)=$ high density with broad, confluent paralesional staining. Care was taken to exclude melanin-containing macrophages (melanophages) as a source of false positive staining. Moreover, distinguishing lymphocyte from DDC staining with HLA-DR was important in avoiding a false positive interpretation; this was readily achieved on morphologic grounds.

\section{Statistical analysis}

Chi-square analysis was performed using Sigma Stat 2.0 software (Jandel Scientific, San Raphael, Ca) run on a Compaq Desk pro 590 PC. Statistical significance was assigned at the $\mathrm{p}<0.05$ level.

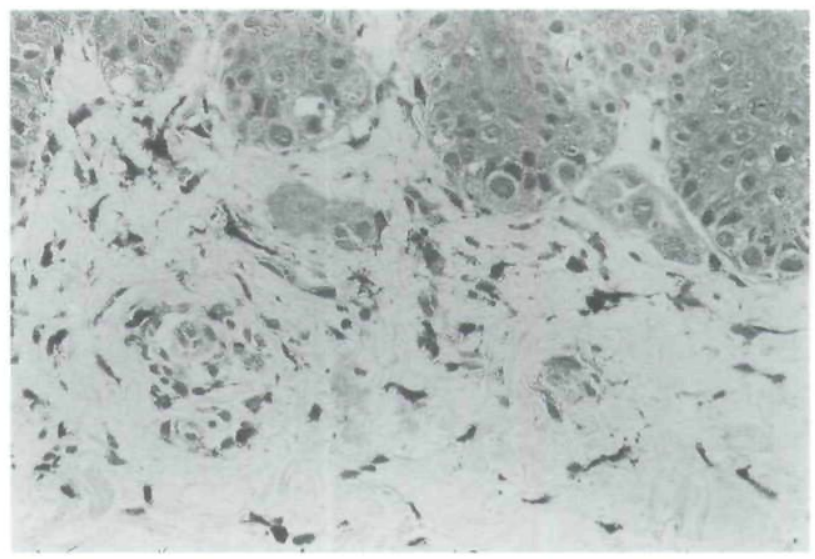

Fig. 1. Slightly increased (1+) factor XIIIa-positive staining of DDCs in a case of radial growth-phase melanoma (Immunoperoxidase, 115×).

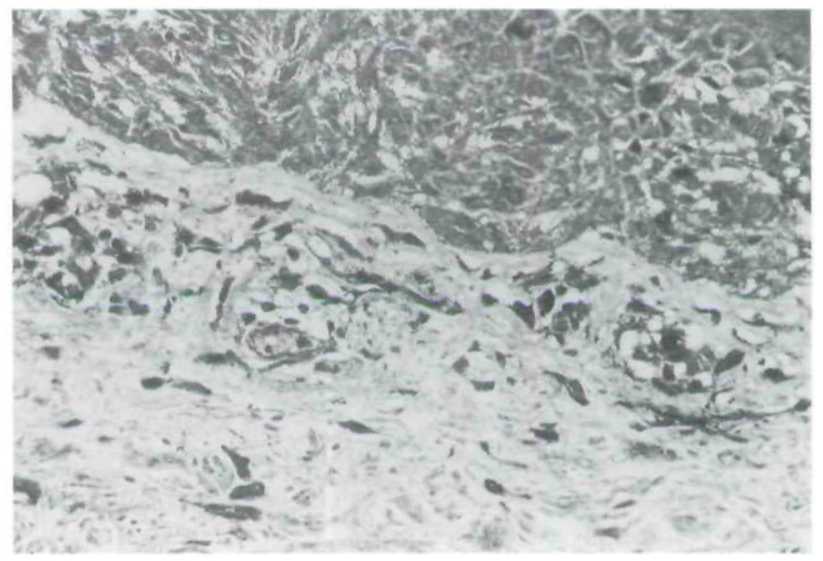

Fig. 2. Slight (1+) HLA-DR (LN3) staining of DDCs in a case of radial growth-phase melanoma (Immunoperoxidase, 115×).

\section{Results}

Factor Xllla staining

Four of $10(40 \%)$ compound nevi, 9 of $20(45 \%)$ radial growth-phase melanomas, and 12 of 20 $(60 \%)$ vertical growth-phase melanomas showed an increase in factor XIIIa staining of DDCs (Table 1). The majority of cases, with the exception of 3 vertical growth-phase melanomas, had only slight (1+) increased staining (Fig. 1). When compared to compound nevi, there was no statistical difference in factor XIIIa staining for radial $(\mathrm{p}=1.00)$ or vertical $(\mathrm{p}=0.44)$ growth-phase melanomas.

\section{HLA-DR staining}

Two of $10(20 \%)$ compound nevi and 17 each of $20(85 \%)$ radial and vertical growth-phase 


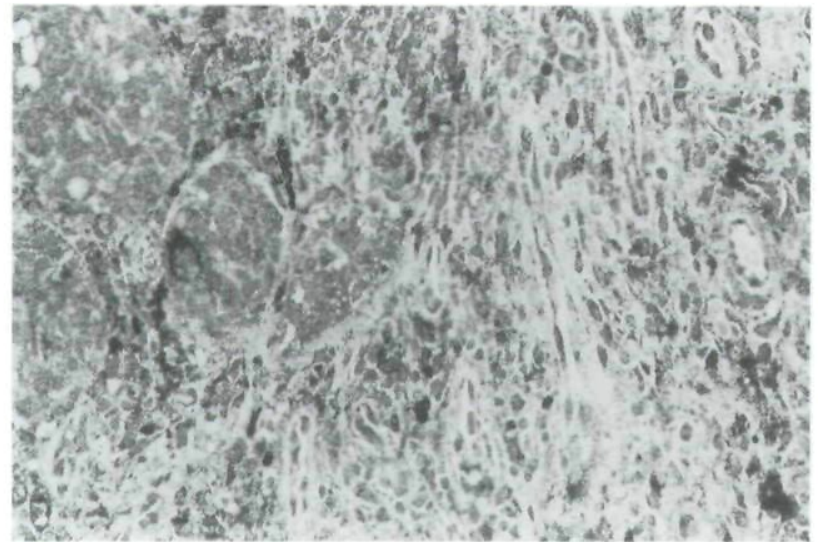

Fig. 3. Moderate (2+) HLA-DR staining of DDCs in a case of vertical growth-phase melanoma. Note the association of positive staining DDCs with the brisk inflammatory lymphocytic infiltrate (Immunoperoxidase, 115x).

melanomas showed HLA-DR expression by DDCs (Table 2). The majority of cases showed slight HLA-DR staining (Fig. 2). However, in 7 of 17 $(41 \%)$ radial and 6 of $17(35 \%)$ vertical growthphase melanomas, the intensity of staining was judged to be moderate (2+) (Fig. 3). HLA-DR expression was judged to be similar in both melanoma groups, but was significantly increased in both melanoma groups when compared to compound nevi $(\mathrm{p}<0.001)$. Of particular interest, HLA-DR expression by DDCs appeared to correlate with the presence or absence of a lymphocytic infiltrate in both radial and vertical growth-phase melanomas. Furthermore, the intensity of staining was greater in the presence of a brisk and infiltrative lymphocytic host response, whereas the intensity of staining was less or absent in a nonbrisk and noninfiltrative or absent lymphocytic host response (Fig. 3).

\section{CD1a staining}

Two of $10(20 \%)$ compound nevi, 3 of $20(15 \%)$ radial growth-phase melanomas, and 5 of $20(25 \%)$ vertical growth-phase melanomas demonstrated slight $(1+)$ increased CDla staining of cells in the superficial dermis (Table 3 ). There was good internal control staining of CDla-positive Langerhans cells within the epidermis. The Langerhans cells within the dermis tended to cluster as a few cells around blood vessels in association with a lymphocytic infiltrate of variable intensity. However, most lesions demonstrating a lymphocytic host response, including both radial and vertical growthphase melanomas, did not show cells staining for $\mathrm{CD}$ la within the dermis. Based on the few cases with CD1a-positive cells in the dermis and the small number of cells that were tightly distributed around blood vessels in a very focal pattern, it is apparent that these cells did not significantly contribute to the HLA-DR expression seen in DDCs.

\section{CD34 staining}

Seven of $10(70 \%)$ and 2 of $10(20 \%)$ compound nevi showed slight (1+) and moderate (2+) CD34 staining, respectively. Eight of $20(40 \%), 6$ of 20 $(30 \%)$, and 3 of $20(15 \%)$ radial growth-phase melanomas showed slight, moderate, and marked CD34 staining, respectively. No vertical growthphase melanomas had marked staining; however, 5 of $20(25 \%)$ had slight and 5 of $20(25 \%)$ had moderate staining (Table 4). DDCs that stained with CD34 had morphologic features indistinguishable from factor XIIIa-positive DDCs, although those CD34-positive DDCs within the interstitium tended to be more evenly distributed from upper to deep dermis. When increased staining was observed, it was usually seen within DDCs along the base or periphery of the lesion. CD34positive DDCs were located both perivascularly and within the dermal interstitium. As expected, CD34 also stained endothelial cells, as well as dendritic cells within the connective tissue surrounding eccrine coils. Since the vascularity varied, in some cases significantly, from case to case, no attempt was made to factor out the contribution of vascular staining to the total score, as reproducibility would be difficult to achieve.

\section{Double labeling}

In 4 of 6 melanoma cases (radial, 2; vertical, 2) on which double labeling for HLA-DR vs. factor XIIIa was performed, frequent factor XIIIa-positive DDCs coexpressing HLA-DR were seen (Fig. 4). There did not appear to be a difference between radial and vertical growth-phase melanomas in number of DDCs staining with both factor XIIIa and HLA-DR. However, there were other cells, mostly lymphocytes but occasional factor XIIIanegative DDCs, expressing only HLA-DR. Occasional factor XIIIa-positive DDCs did not show HLA-DR coexpression. Two cases showed strong HLA-DR staining but very weak and patchy factor XIIIa staining; these cases were considered unreliable for interpretation. It is possible that antigen retrieval for factor XIIIa by microwaving was suboptimal on these two cases.

When CD34 and factor XIIIa were applied in our double labeling technique, DDCs were readily identified with restricted staining for either CD34 or factor XIIIa in 3 of 6 cases (Fig. 5). No DDCs coexpressing both epitopes were seen. In 3 of 6 cases, CD34 stained DDCs well; however, factor XIIIa 


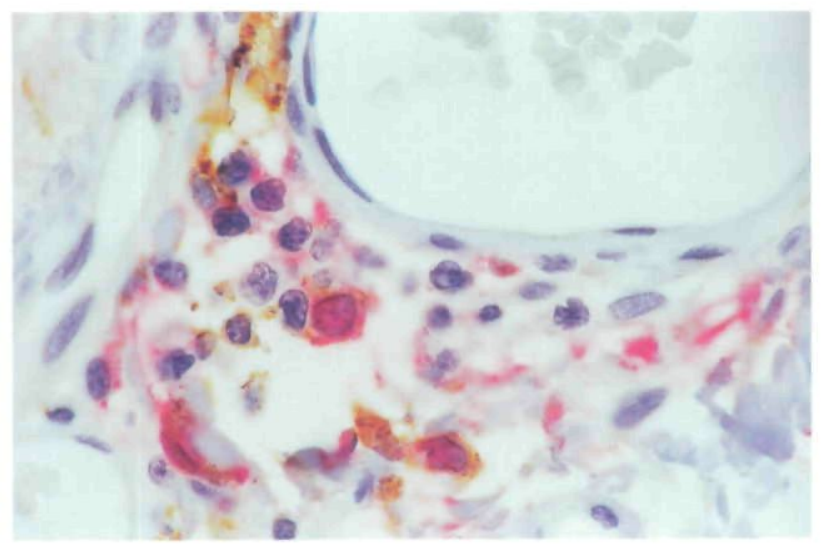

Fig. 4. Perivascular factor XIIIa-positive (red) DDCs demonstrating coexpression of HLA-DR (brown) by double labeling (Immunoperoxidase, 825x).

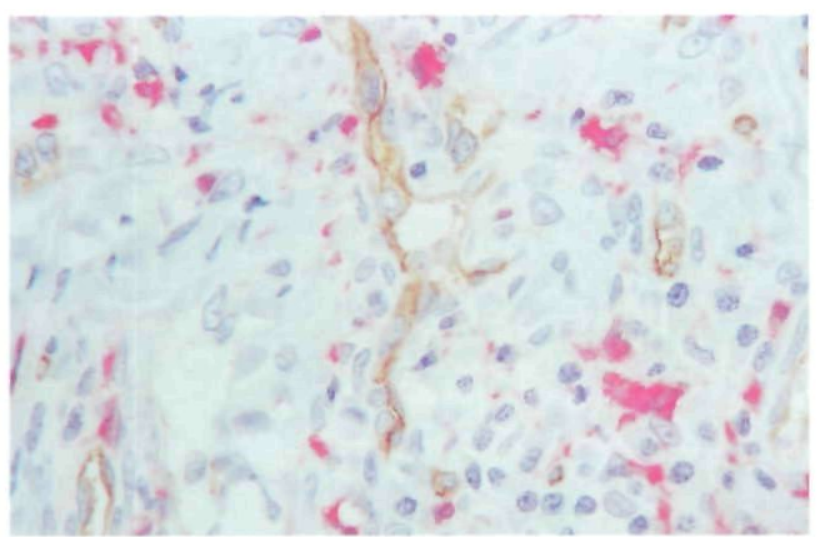

Fig. 5. Restricted staining of CD34 (brown) within endothelial cells and occasional hematopoietic cells, and factor XIIIa (red) within DDCs (Immunoperoxidase, 528x).

staining was weak and focal. Therefore, as with a few cases of HLA-DR vs. factor XIIIa, these cases were considered unreliable for interpretation.

CD34 was stained against HLA-DR to see if any CD34-positive DDCs may coexpress HLA-DR, possibly accounting for the occasional DDCs expressing HLA-DR that were factor XIIIa-negative. Five of 6 cases showed strong staining for both HLADR and CD34. No CD34 DDCs were observed to coexpress HLA-DR. In one case, HLA-DR staining was strong but CD34 staining was absent. Therefore, this case was considered unreliable for interpretation.

\section{Discussion}

In contrast to fibroproliferative lesions, such as dermatofibromas or fibrous papules of the face, there was no significant hyperplasia of DDCs, as determined by cytoplasmic factor XIIIa labeling, in the nevomelanocytic lesions we evaluated. Our
Table 3. Results of CD1a staining of cells within the dermis.

\begin{tabular}{lcccc}
\hline Diagnosis & \multicolumn{4}{c}{ Intensity of staining } \\
\cline { 2 - 5 } & 0 & $1+$ & $2+$ & $3+$ \\
\hline Compound nevi & 8 & 2 & 0 & 0 \\
Radial growth-phase melanomas & 17 & 3 & 0 & 0 \\
Vertical growth-phase melanomas & 15 & 5 & 0 & 0 \\
\hline
\end{tabular}

Table 4. Results of CD34 staining of DDCs.

\begin{tabular}{lcccc}
\hline Diagnosis & \multicolumn{4}{c}{ Intensity of staining } \\
\cline { 2 - 5 } & 0 & $1+$ & $2+$ & $3+$ \\
\hline Compound nevi & 1 & 7 & 2 & 0 \\
Radial growth-phase melanomas & 3 & 8 & 6 & 3 \\
Vertical growth-phase melanomas & 10 & 5 & 5 & 0 \\
\hline
\end{tabular}

postulate that DDCs would be increased above background in invasive melanomas when compared to a benign nevomelanocytic control group was not confirmed in this study. Moreover, if DDCs increased in invasive melanomas, either due to proliferation or recruitment, we hypothesized that the number of DDCs would be either unchanged or, more likely, reduced in vertical growth-phase melanomas when compared to radial growthphase melanomas. We found no difference in the number of factor XIIIa-positive DDCs between vertical and radial growth-phase melanomas, as determined semiquantitatively.

Radial and vertical growth-phase melanomas are associated with a significantly increased expression of HLA-DR by DDCs in single antibody immunoperoxidase studies, suggesting that DDCs may have an antigen-presenting role in the dermal immune response to invasive melanomas. This is perhaps not surprising, since HLA-DR expression by DDCs in intradermal delayed hypersensitivity reactions has been described (1). In order to confirm that factor XIIIa-positive cells may coexpress HLA-DR, we subjected a small subset of radial and vertical growth-phase melanomas to an immunoperoxidase double labeling technique. We found that some factor XIIIa-positive DDCs coexpress HLA-DR (cells with cytoplasmic staining for both antigens), thereby confirming our impression from single antibody studies. We acknowledge the remote possibility of cross reactivity in our double labeling experiment, since the secondary antibody used against both primary antibodies was polyspecific. However, based on the pattern of DDCs staining for HLA-DR and factor XIIIa, i.e. coexpression as well as cells with restricted staining with both antibodies, purely restricted staining within DDCs for either CD34 or factor XIIIa, and cells with either CD34 or HLA-DR expression, we are confident that all of the binding sites on the 
initial polyspecific secondary antibody were adequately blocked by the avidin-biotin amplification system such that no sites were available for cross reactivity.

In most radial and vertical growth-phase melanomas, HLA-DR expressing DDCs were found in association with the host lymphocytic inflammatory response and, more importantly, appeared to be associated with a brisk and infiltrative lymphocytic response as described by Elder and coworkers $(22,23)$. This finding may further support a putative antigen-presenting role by DDCs to tumor infiltrating lymphocytes.

The DDC remains an under studied cell in the dermal immune response to the wide range of inflammatory and neoplastic processes that involve the skin. Additional studies of HLA-DR-expressing DDCs in melanomas with a brisk and infiltrative lymphocytic host response compared to melanomas with a brisk and noninfiltrative, nonbrisk, or absent lymphocytic host response should be done.

\section{Acknowledgments}

We thank Dr. Kirk Wojno and Dr. Timothy Singleton in the Department of Pathology at the University of Michigan Medical Center for their technical expertise provided in immunoperoxidase staining. We also thank Rong Sun in the Research Laboratory of Dr. Timothy Singleton, and Tina Fields and Kathy Smieszny in the Immunoperoxidase Laboratory at the University of Michigan Medical Center for their time and technical expertise provided in performing the immunoperoxidase stains on the cases in this series.

This work was presented in part as an abstract at the United States and Canadian Academy of Pathology, March 4, 1997, Orlando, Fl.

\section{References}

1. Headington, JT. The dermal dendrocyte. In: Callen JP, Dahl MV, Goletz LE et al., eds. Advances in Dermatology. Chicago: Year Book Medical Publisher Inc., Vol. 1. 1986: 159 .

2. Sontheimer RD, Matsubara T, Seelig LL. A macrophage phenotype for a constitutive, class II antigen-presenting, human dermal perivascular dendritic cell. J Invest Dermatol 1989: 93: 154.

3. Cerio R, Griffiths CE, Cooper KD, Nickoloff BJ, Headington JT. Characterization of factor XIIIa positive dermal dendritic cells in normal and inflamed skin. Br J Dermatol 1989: 121: 421.

4. Estrada JA, Piérard GE. Factor-XIIIa-positive dendrocytes and the dermal microvascular unit. Dermatologica 1990: 180: 51 .

5. Derrick EK, Barker JN, Khan A, Price ML, MacDonald DM. The tissue distribution of factor XIIIa positive cells.
Histopathology 1993: 22: 157.

6. Cerio R, Spaull J, Wilson Jones E. Identification of factor XIIIa in cutaneous tissue [Letter]. Histopathology 1988: 13: 362 .

7. Headington JT, Cerio R. Dendritic cells and the dermis. Am J Dermatopathol 1990: 12: 217.

8. Cerio R, Spaull J, Wilson Jones E. Histiocytoma cutis: a tumour of dermal dendrocytes (dermal dendrocytoma). $\mathrm{Br}$ J Dermatol 1989: 120: 197 .

9. Nemeth AJ, Penneys NS, Bernstein HB. Fibrous papule: a tumor of fibrohistiocytic cells that contain factor XIIIa. J Am Acad Dermatol 1988: 19 : 1102.

10. Gray MH, Trimble CL, Zirn J, McNutt NS, Smoller BR, Varghese M. Relationship of factor XIIIa-positive dermal dendrocytes to Kaposi's sarcoma. Arch Pathol Lab Med 1991: 115: 791.

11. Denton KJ, Cotton DW, Wright A, Hird P. Factor XIIIa in nodular malignant melanoma and Spitz naevi. Br J Dermatol 1990: 123: 783 .

12. Nestle FO, Zheng XG, Thompson CB, Turka LA, Nickoloff BJ. Characterization of dermal dendritic cells obtained from normal human skin reveals phenotypic and functionally distinctive subsets. J Immunol 1993: 151: 6535 .

13. Ruiter DJ, Bhan AK, Harrist TJ, Sober AJ, Mihm MC. Major histocompatibility antigens and mononuclear inflammatory infiltrate in benign nevomelanocytic proliferations and malignant melanoma. J Immunol 1982: 129: 2808.

14. van Vreeswijk H, Ruiter DJ, Bröcker EB, Welvaart K, Ferrone S. Differential expression of HLA-DR, DQ and DP antigens in primary and metastatic melanoma. J Invest Dermatol 1988: 90: 755.

15. Zaloudik J, Moore M, Ghosh AK, Mechl Z, Rejthar A. DNA content and MHC class II antigen expression in malignant melanoma: clinical course. J Clin Pathol 1988: 41: 1078.

16. Becker JC, Bröcker EB. Lymphocyte melanoma interaction: role of surface molecules. Recent Results Cancer Res 1995: 139: 205.

17. Bröcker EB, Suter L, Sorg C. HLA-DR antigen expression in primary melanomas of the skin. J Invest Dermatol 1984: 82: 244.

18. Holtzmann B, Bröcker EB, Lehman JM, et al. Tumor progression in human melanoma: five stages defined by their antigenic phenotypes. Int J Cancer 1987: 39: 466.

19. Brogelli L, Moretti S, Giannotti B. Superficial spreading melanoma with a nodular area: antigenic phenotype of the radial and vertical growth areas. Tumori 1989: 75: 8 .

20. Toriyama K, Wen DR, Paul E, Cochran AJ. Variations in the distribution, frequency, and phenotype of Langerhans cells during the evolution of malignant melanoma of the skin. J Invest Dermatol 1993: 100: 269s.

21. Garcia-Plata D, Lessana-Leibowitch M, Palangie A, et al. Immunophenotype analysis of dendritic cells and lymphocytes associated with cutaneous malignant melanomas. Invasion Metastasis 1995: 15: 125.

22. Elder DE, Guerry D IV, Van Horn M, et al. The role of lymph node dissection for clinical stage I malignant melanoma of intermediate thickness (1.51-3.99 mm). Cancer 1985: 56: 413.

23. Clark WH Jr, Elder DE, Guerry D IV, et al. Model predicting survival in stage I melanoma based on tumor progression. J Natl Cancer Inst 1989: 81: 1893. 
This document is a scanned copy of a printed document. No warranty is given about the accuracy of the copy. Users should refer to the original published version of the material. 